\title{
Application of Etiology List in Differential Diagnosis of Acute Abdominal Pain*
}

\author{
Chang Lv' ${ }^{1}$ Xurui Li ${ }^{1}$, Zhichao Ma', Cuiya Wang², Junna Zhang1, Wei Sun', \\ Zhantao Jiao', Jianguo Li'"\#
}

${ }^{1}$ Hebei General Hospital, Shijiazhuang, China

${ }^{2}$ Graduate School of Hebei Medical University, Shijiazhuang, China

Email: "lijg65@163.com

How to cite this paper: Lv, C., Li, X.R., Ma, Z.C., Wang, C.Y., Zhang, J.N., Sun, W., Jiao, Z.T. and Li, J.G. (2021) Application of Etiology List in Differential Diagnosis of Acute Abdominal Pain. Open Journal of Emergency Medicine, 9, 40-47.

https://doi.org/10.4236/ojem.2021.92006

Received: May 17, 2021

Accepted: June 12, 2021

Published: June 15, 2021

Copyright $\odot 2021$ by author(s) and Scientific Research Publishing Inc. This work is licensed under the Creative Commons Attribution International License (CC BY 4.0).

http://creativecommons.org/licenses/by/4.0/

\begin{abstract}
Objective: To use the etiology list of abdominal pain to help identify acute abdominal pain. Methods: to design a list of causes of acute abdominal pain as per the concept of system thinking, determine the five differential diagnosis directions of "local organ disease, adjacent organ disease, systemic disease, psychogenic disease, and female reproductive system disease", and elaborate the application effect of the checklist in the diagnosis of acute abdominal pain by virtue of the analysis of one case of acute abdominal pain. Results: according to the list of causes of abdominal pain, the causes of acute abdominal pain were screened, and patients suffering from acute renal infarction (ARI) who presented with simple abdominal pain were diagnosed and treated timely. Conclusion: the etiology list is helpful to guide the diagnosis direction of acute abdominal pain, and make a rapid clinical diagnosis, so as to form a systematic thinking mode.
\end{abstract}

\section{Keywords}

Cbdominal Pain, Check List, Clinical Decision, Acute Renal Infarction (ARI)

\section{Introduction}

Abdominal pain, one of the common symptoms in the Emergency Department, has complex etiological factors, usually involving internal medicine, surgical, pediatric, and gynecological diseases [1], with sudden onset and rapid changes. The complexity of the etiology of abdominal pain makes emergency physicians really confused, and it is a very heavy task for any of them to remember and master the differential diagnosis of abdominal pain, thereby falling into a wrong

${ }^{*}$ New thinking for differential diagnosis of acute abdominal pain.

${ }^{*}$ Corresponding author. 
path [2]. It is even more challenging for emergency physicians who are jammed for time. How to deal with this challenge has always been a difficulty for emergency physicians. This study, based on the concept of system thinking, and following the principle of "simplicity first" in the Checklist [3], reclassifies the causes of abdominal pain, establishes an etiology list of abdominal pain, grasps the overall and integral diagnosis of abdominal pain, screens the causes of abdominal pain and quickly diagnoses cases of renal artery infarction with abdominal pain. The purpose of this study is to broaden the lateral thinking of emergency physicians on the diagnosis of abdominal pain and to form a thinking mode to improve the differential diagnosis of abdominal pain and reduce misdiagnosis and missed diagnosis. This study is approved by the Hebei General Hospital Ethics Committee, and for the case to be introduced, the Informed Consent Form has been signed with the family members.

\section{Method}

An application of an etiology list of abdominal pain: based on the concept of system thinking and anatomical location, and following the principle of integrity of clinical thinking [4], an etiology list of abdominal pain in five key directions of "local organ disease, adjacent organ disease, systemic disease, psychogenic disease, and female reproductive system disease" was designed [5] (see Table 1), as the top layer of the modular differential diagnosis program. In the second and third layers, the anatomical location and lesions natures were used to define subcategories. The secondary classification of local organ diseases includes abdominal and abdominal wall diseases; the adjacent organ diseases include respiratory, mediastinal, and spinal diseases; By analogy, the systemic diseases include blood diseases, endocrine metabolism, immunity, poisoning diseases, etc. In the fourth layer of the program, the common and frequently occurring diseases of diagnostics are embraced to rank the types of diseases that cause abdominal pain. Then in the light of the step-down thinking for screening [4], Then according to the list of causes of abdominal pain, the direction of diagnostic thinking was guided, the differential diagnosis of abdominal pain is improved.

\section{Clinical Application}

Abdominal pain is easy to be misdiagnosed and omitted in the early stage, leading to improper treatment which may endanger the life of patients. In particular, for abdominal vascular diseases, such as acute renal infarction, some patients may have no symptoms or signs, lacking specific clinical manifestations and laboratory examinations [6]. The following describes the application ideas and effects of the etiology list in acute renal infarction.

\subsection{Case Report}

Female, 77 years old. Chief complaint: abdominal pain for 2 days. Two days ago, the patient suffered from the persistent dull pain in the left lower abdomen, which was mainly in lower abdomen, and had nothing to do with the activity or 
Table 1. Etiology list of abdominal pain.

\begin{tabular}{|c|c|c|}
\hline Anatomical orientation & $\begin{array}{l}\text { Etiological } \\
\text { classification }\end{array}$ & Acute common diseases \\
\hline \multirow[t]{4}{*}{ Peritoneal cavity } & Inflammation & $\begin{array}{l}\text { Gastritis, enteritis, pancreatitis, } \\
\text { hemorrhagic necrotizing enteritis, } \\
\text { cholecystitis, purulent obstructive } \\
\text { cholecystitis, etc.; reflux esophagitis, } \\
\text { spontaneous peritonitis; urinary } \\
\text { tract infection. }\end{array}$ \\
\hline & Viscera rupture & $\begin{array}{l}\text { Liver rupture, spleen rupture, etc.; } \\
\text { perforation of stomach and duodenal ulcer. }\end{array}$ \\
\hline & $\begin{array}{l}\text { Obstruction of } \\
\text { hollow viscera }\end{array}$ & $\begin{array}{l}\text { Intestinal obstruction, biliary stone, biliary } \\
\text { ascariasis, obstruction of kidney and ureter } \\
\text { stones, etc.; volvulus, mesentery or torsion } \\
\text { of the omentum }\end{array}$ \\
\hline & $\begin{array}{l}\text { Intra-abdominal } \\
\text { vascular lesion }\end{array}$ & $\begin{array}{l}\text { Mesenteric artery embolism, splenic } \\
\text { embolism, renal embolism, aneurysm of } \\
\text { abdominal acute renal infarction, etc. }\end{array}$ \\
\hline Abdominal wall & Abdominal wall & $\begin{array}{l}\text { Abdominal wall diseases such as abdominal } \\
\text { wall contusion, abscess, and herpes zoster } \\
\text { on the abdominal wall }\end{array}$ \\
\hline 2) Adjacent & $\begin{array}{l}\text { Cardiopulmonary } \\
\text { diseases and } \\
\text { spinal lesions }\end{array}$ & $\begin{array}{l}\text { Angina, myocardial infarction, acute } \\
\text { pericarditis, pneumonia, URTI, pulmonary } \\
\text { infarction, pleurisy, hiatus hernia, thoracic } \\
\text { vertebrae tuberculosis or tumor }\end{array}$ \\
\hline 3) Systemic & Systemic diseases & $\begin{array}{l}\text { Such as abdominal allergic purpura, } \\
\text { abdominal epilepsy, uremia, } \\
\text { hematoporphyria, SLE, and poisoning. }\end{array}$ \\
\hline 4) Psychogenic & Neurosis & Abdominal pain neurosis \\
\hline 5) Female reproductive system & $\begin{array}{l}\text { Gynecological } \\
\text { disease }\end{array}$ & $\begin{array}{l}\text { Ectopic pregnancy and ovarian rupture, } \\
\text { ovarian cyst torsion, and ovarian bleeding }\end{array}$ \\
\hline
\end{tabular}

body position. It was accompanied by nausea, and no vomiting, radiating pain, stopping flatus and defecation, fever and chills, chest tightness and chest pain, cough and sputum, and other simultaneous phenomena. She went to a local hospital for examination, and the results showed that she suffers from atrial fibrillation, high hemogram, slightly high D-dimer. She took painkillers and spasmolysis medicines, but the effect was unsatisfactory. Later, she was transferred into our hospital. Past history: physical examinations: T $37.2^{\circ} \mathrm{C}, \mathrm{P} 123$ beats/min, R16 beats/min, BP 114/77 mmHg, $\mathrm{SPO}_{2} 96 \%$, VAS (Visual Analogue Scale) pain score: 6 points (0 point: no pain; 3 points or less: slight pain; tolerable; 4 - 6 points: pain; affecting sleep, still tolerable; $7-10$ points: strong pain; unbearable, affecting appetite and sleep). Consciousness, low spirit, pupils with equal sizes and circles, light reflection, there is no abnormality in the peripheral skin, acute illness, clear breath sounds in the both lungs, no dry and wet rales, arrhythmia and no murmur in any valve area. Moderate tightness of the abdomen, pressing pain in the left upper abdomen and lower abdomen, no rebound tenderness and muscular tension, the liver and spleen not be detected, and nor- 
mal bowel sounds. Percussion pain (negative) for both kidneys; percussion pain (negative) for spines; no edema found in both lower extremities. ECG: atrial fibrillation with rapid ventricular rate; Troponin T: $<40 \mathrm{ng} / \mathrm{L}(0-100 \mathrm{ng} / \mathrm{L}) ;$ blood gas analysis: $\mathrm{PH}$ value; 7.48 (7.35 - 7.45); $\mathrm{PCO}_{2}: 29.20 \mathrm{mmHg}(35$ - $45 \mathrm{mmHg}$ ); $\mathrm{PO}_{2}: 88.70 \mathrm{mmHg}\left(80\right.$ - $100 \mathrm{mmHg}$ ); $\mathrm{SO}_{2}: 97.10 \%$ (95.0\% - 98.0\%); lactic acid: $2.30 \mathrm{mmol} / \mathrm{L}(1.0-1.7 \mathrm{mmol} / \mathrm{L})$; blood analysis: WBC $14.67 \times 10^{9} / \mathrm{L}(3.5-9.5 \times$ $10 \% / \mathrm{L}) ; \mathrm{N} 85.60 \%$ (40\% - 75\%); HGB $130.00 \mathrm{~g} / \mathrm{L}(115-150 \mathrm{~g} / \mathrm{L}) ;$ PLT $198.00 \times$ $10^{9} / \mathrm{L}\left(125-350 \times 10^{9} / \mathrm{L}\right)$; Nine items of examination for emergency treatment: total protein $66.0 \mathrm{~g} / \mathrm{L}(65-85 \mathrm{~g} / \mathrm{L})$; kalium $4.1 \mathrm{mmol} / \mathrm{L}(3.5-5.3 \mathrm{mmol} / \mathrm{L})$; sodium $127 \mathrm{mmol} / \mathrm{L}$ (137 - $147 \mathrm{mmol} / \mathrm{L})$; chlorine $97 \mathrm{mmol} / \mathrm{L}$ (99 - $110 \mathrm{mmol} / \mathrm{L})$; carbon dioxide combining power $18.7 \mathrm{mmol} / \mathrm{L}$ ( 22 - $29 \mathrm{mmol} / \mathrm{L})$; creatinine 93 umol/L (41 - $81 \mathrm{umol} / \mathrm{L})$; glucose: $5.94 \mathrm{mmol} / \mathrm{L}$ (3.9 - $6.1 \mathrm{mmol} / \mathrm{L})$; no abnormal urine routine; five items of examination for blood coagulation: D-dimer 1.12 mg/L FEU (0 - 0.55 mg/L FEU); PT: $12.1 \mathrm{~s}$ (9.8 - $12.1 \mathrm{~s})$; international normalized prothrombin ratio: 1.05 (0.85 - 1.3); FIB: $3.2 \mathrm{~g} / \mathrm{L}(2-4 \mathrm{~g} / \mathrm{L})$; pancreatin determination: amylase: $72 \mathrm{U} / \mathrm{L}(35-135 \mathrm{U} / \mathrm{L})$.

\subsection{Case Analysis}

Among the causes of abdominal pain, the patient, female, 77 years old, has been menopausal for 20 years. Therefore, the fertility-related diseases of the female reproductive system are excluded; the patient felt moderate tightness of the abdomen, no fixed-point tenderness, and abdominal wall diseases are not supported, which can be excluded; there were no fever and chills, rebound tenderness and muscular tension, abdominal distension, and stopping flatus and defecation, percussion pain (negative) for both kidneys, WBC $14.67 \times 10^{9} / \mathrm{L}$ (reference range $3.5-9.5 \times 10^{9} / \mathrm{L}$ ); N 85.60\% (reference range $40 \%-75 \%$ ), local diseases such as acute inflammation, viscera rupture, torsion and obstruction of hollow viscera not supported; the patient had atrial fibrillation with rapid ventricular rate, severe abdominal pain, D-dimer $1.12 \mathrm{mg} / \mathrm{L}$ FEU (reference range 0 - $0.55 \mathrm{mg} / \mathrm{L} \mathrm{FEU}$ ), and took spasmolysis medicines, but the effect was unsatisfactory. The intra-abdominal vascular lesion is to be excluded. However, the patient's abdominal pain lasted for 2 days, and there were no symptoms and signs of intestinal necrosis, and it was difficult to explain with common mesenteric artery embolism. Perhaps the symptoms and signs of the elderly can be atypical, except for observation and further examination; the patient had no chest tightness and chest pain, cough and sputum, with clear breath sounds in both lungs, and no dry and wet rales. Percussion pain (negative) for spines, ECG (-), Troponin T: $<40 \mathrm{ng} / \mathrm{L}(0$ - $100 \mathrm{ng} / \mathrm{L})$, adjacent viscera diseases such as cardiopulmonary diseases and spinal lesions not supported, which remains to be observed. Blood gas analysis, electrolytes, normal liver and kidney functions and blood sugar, no underlying diseases and medication history; abdominal pain caused by systemic diseases is not considered temporarily; VAS (Visual Analogue Scale) pain score: 6 points, consciousness, low spirit, face of acute ill; psychogenic diseases can be excluded; In conclusion: the patient had abdominal pain for 2 days 
and was transferred to the hospital without any other discomfort. Upon the analysis of the list, the abdominal pain caused by intra-abdominal vascular lesion cannot be excluded. If the disease continues to worsen, it may be lifethreatening. We directly performed enhanced CT of the abdomen and pelvis, and the results showed: the main trunk of the left renal artery is thinner than that of the right renal artery. The thrombosis in the distal branch lumen of the left renal artery is more likely to cause large-area infarction of the left kidney. The mesenteric vessels are well developed without embolism. The patient was admitted to the Vascular Surgery Department with definitive diagnosis.

\section{Discussion}

Diagnosis is a complex cognitive process covering a number of links [7]. Currently, the thinking mode commonly used in emergency treatment is hypothetical deduction [8], that is, based on the patient's symptoms, and in combination with medical history, detailed physical examination and necessary laboratory examination, first, a certain disease is assumed, and then further deterministic inspections are performed, to compare the results obtained with the hypothetical disease characteristics, and judge whether the hypothesis is true, so as to obtain a preliminary diagnosis, which belongs to the model-controlled diagnosis mode [7]. The decision-making process relies on personal experience. It is more advantaged over rapid diagnosis for common diseases, but it is very difficult to diagnose complex or rare diseases. Since people are concerned about common and frequently-occurring diseases, or often ignore rare diseases due to lack of experience, misdiagnosis and missed diagnosis are easily caused since they can't think of or don't think about it [2].

This study reorganizes the causes of acute abdominal pain, reclassifies the causes of abdominal pain, and designs five key diagnostic directions of "local organ disease, adjacent organ disease, systemic disease, psychogenic disease, and female reproductive system disease", which covers all common causes of abdominal pain, grasps the differential diagnosis of abdominal pain as a whole, and forms a systematic thinking mode.

This study reorganizes the causes of acute abdominal pain, reclassifies the causes of abdominal pain, and designs five key diagnostic directions of "local organ disease, adjacent organ disease, systemic disease, psychogenic disease, and female reproductive system disease", which covers all common causes of abdominal pain, grasps the differential diagnosis of abdominal pain as a whole, and forms a systematic thinking mode.

The cause of abdominal pain is complex, and it is difficult to fully grasp the differential diagnosis of abdominal pain. As a result, the misdiagnosis rate is fairly high [2] [9], especially among the elderly. It has been shown that: the misdiagnosis rate in the emergency room for patients $\geq 65$ years old is $52 \%$ [10]. Acute renal infarction is mainly manifested by abdominal pain, and it has been reported that flank pain in patients with acute renal infarction accounts for 
$71.2 \%$ [11]. It is a rare disease with an incidence of $0.004 \%$ of emergency patients [12]. Hematuria is of suggestive significance for the diagnosis of ARI, but it is not common. It is neither a sensitive diagnostic clue, nor a specific clinical manifestation, and it makes the diagnosis of acute renal infarction more difficult [10]. Not every acute renal infarction will develop the symptoms described in the textbook. Even if these symptoms are developed, because the symptoms overlap with other diseases, the symptoms of different diseases are the same. As a result, it's not easy to make a diagnosis because it can't be associated with acute renal infarction by only one or several symptoms developed. In the meantime, the understanding of symptoms requires the accumulation of experience. Any experience cannot form a fixed thinking mode and method for most physicians. It is difficult for clinicians to integrate all the causes of abdominal pain in a short period of time, and unable to conduct comprehensive screening for the causes of abdominal pain. In general, explaining the knowledge structure of disease diagnosis requires years of learning and experience, and only experts manage to establish a differential diagnosis from symptoms to diseases in a relatively short period of time [13].

This study, oriented towards abdominal pain, establishes a relatively complete etiology list of abdominal pain from symptoms to diseases. It is the same as the diagnosis model established by the School of Medicine, University of Calgary [14]. It quickly builds a thinking mode of experts, which not only gives directions of medical history, physical examination, and laboratory examination, but also collects the clinical data of the patient, and screens the diseases in the list one by one, without missing key diseases. As for the case of this study, the patient presented with abdominal pain and suffered from persistent dull pain in the left lower abdomen. In this case, it is difficult for physicians to think of acute renal infarction, and the next step of examination and diagnosis is blind. According to the list, the four aspects of "adjacent organ disease, systemic disease, psychogenic disease, and female reproductive system disease" are excluded via medical history, symptom physical examination, and general laboratory examination. In local organ diseases, the patient did not suffer from "fever, obvious tenderness and rebound tenderness", and viscera rupture, torsion, and obstruction of hollow viscera are not supported. Although the symptoms and signs of the elderly can be atypical, except for observation and further examination, the list suggests: "Vascular abdominal pain" is a life-threatening disease that needs to be ruled out. Therefore, we directly performed angiography and did not carry out CT abdominal plain scan, thus saving the diagnosis time and finally clarifying the diagnosis.

The etiology list of abdominal pain is simple and easy to remember, in which checking points are clearly set to guide the diagnosis of abdominal pain and broaden the physician's lateral thinking. In regard to the effectiveness of the "list", Atul Gawande described in detail in his book "The Checklist Manifesto". Johns Hopkins Hospital, by virtue of a small checklist, reduced the percentage of 
central venous catheter infections to 0 from 11\%; after 15 months, 43 infections and 8 fatal accidents were avoided, helping the hospital save USD 2 million of the cost [3]. The "list of thinking" is in conformity with the principle of mind mapping, but it is more concise, helping us forese rare diseases, and gives the rapid diagnosis, thus avoiding misdiagnosis and missed diagnosis caused by atypical clinical manifestations and rare diseases.

The list is not under a blind screening but based on the prompts of the list to screen for suspicious diseases, which is also well-targeted. If no possible diseases are found, a second screening will be conducted as per the list from 5 directions, without missing key diseases just as the first screening, which conforms to the principle of critical thinking.

The shortcomings of this study are as follows: due to the simple operation of the diagnosis process, the physician may develop menu-based thinking and the possibility of excessive medical care for inexperienced physicians. Therefore, the long-term effect of this program needs to be further observed.

\section{Authors' Contributions}

Chang Lv wrote this paper; Xurui Li, Zhangshun Shen, and Cuiya Wang consulted data and wrote revisions; Jianguo Li carried out the conception of this paper. This paper has no conflict of interest.

\section{Funding}

2020 Medical Science Research Project Plan of Hebei Province 20200014.

\section{Conflicts of Interest}

The authors declare no conflicts of interest regarding the publication of this paper.

\section{References}

[1] GAO, Y.X., LI, L., LI, Y., et al. (2014) Diferential Diagnosis of 1533 Cases NonTraumatic Acute Abdominal Pain. Journal of Clinical Emergency, 15, 315-318.

[2] Samantha, Y., Chen, L., Wesley, P., Wong, P. and Wong, J. (2020) Led Astray. New England Journal of Medicine, 383, 578-583. https://doi.org/10.1056/NEJMcps1900799

[3] Gawande, A. (2017) The Checklist Manifesto: How to Get Thinks Right. Beijing union Press, Beijing, 10, 46-56.

[4] Zhao, Q., Guo, H., Shen, Z.S., et al. (2019) On the Nature and Principles of Clinical Thinking. Medicine \& Philosophy, 40, 15-19.

[5] Pan, H.M., Li, H.L., Shen, Z.S., Guo, H., Zhao, Q. and Li, J.G. (2021) Observation of the Effectiveness of a Diagnostic Model for Acute Abdominal Pain Based on the Etiology Checklist and Process Thinking. Risk Management and Healthcare Policy, 14, 835-845. https://doi.org/10.2147/RMHP.S295142

[6] Ma, Q., Liu, D.C. and Yu, J. (2013) Diagnosis of Ureteral Calculi with Acute Renal Infarction. Report Misdiagnosis, 26, 41-42. 
[7] Wu, D. (2015) Clinical Thinking and Its Dynamic Characteristics. Chinese Journal of Diagnostics (Electronic Edition), 3, 90-97.

[8] Tibbles, A. (1992) Developing Clinical Problem-Solving Skills A Guide to More Effective Diagnosis and Treatment. Journal of the Canadian Chiropractic Association, $36,173$.

[9] Dagiely, S. (2006) An Algorithm for Triaging Commonly Missed Causes of Acute Abdominal Pain. Journal of Emergency Nursing, 32, 91-93. https://doi.org/10.1016/j.jen.2005.09.014

[10] Laurell, H., Hansson, L.E. and Gunnarsson, U. (2006) Diagnostic Pitfalls and Accuracy of Diagnosis in Acute Abdominal Pain. Scandinavian Journal of Gastroenterology, 41, 1126-1131. https://doi.org/10.1080/00365520600587485

[11] Zhang, Z.-G. and Liu, X.-M. (2019) Clillical Characteristics of Patients with Acute Renal Inflarction: An Analysis of 52 Patients in a Single Center. Journal of Peking University (Health Sciences), 51, 863-869.

[12] Huang, C.C., Kao, W.F., Yen, D.H., et al. (2006) Renal Infarction withou the Maturia: Two Case Reports. Journal of Emergency Medicine, 30, 57-61. https://doi.org/10.1016/j.jemermed.2005.03.013

[13] (2012) Developing a Clinical Presentation Curriculum in Veterinary Education: A Cognitive Perspective. Comparative Clinical Pathology, 21, 1521-1526. https://doi.org/10.1007/s00580-011-1323-3

[14] Mandin, H., Harasym, P., Eagle, C., et al. (1995) Developing a "Clinical Presentation" Curriculum at the University of Calgary. Academic Medicine, 70, 186-193. https://doi.org/10.1097/00001888-199503000-00008 